\title{
A technical report from the Italian SARS-CoV-2 outbreak. Postmortem sampling and autopsy investigation in cases of suspected or probable COVID-19
}

\author{
Alessandro Santurro ${ }^{1} \cdot$ Matteo Scopetti $^{1} \cdot$ Stefano D'Errico $^{2} \cdot$ Vittorio Fineschi $^{1}$ (D) \\ Accepted: 24 April 2020 / Published online: 12 May 2020 \\ (C) Springer Science+Business Media, LLC, part of Springer Nature 2020
}

\begin{abstract}
SARS-CoV-2 (severe acute respiratory syndrome coronavirus 2) is a coronavirus responsible for COVID-19 (coronavirus disease 2019) which resulted in a cluster of cases of pneumonia that originated in China around 31 December 2019 and has subsequently spread across the globe. Currently, COVID-19 represents a health emergency worldwide, leading, in severe cases, to pneumonia, severe acute respiratory syndrome, multiorgan dysfunction or failure, and death. In the context of limited scientific knowledge and evidence of SARS-CoV-2 infection, guidance is becoming increasingly necessary for pathologists who have to perform postmortem investigations on COVID-19 cases. The aim of the present report is to share a procedure applicable to cases of COVID-19-related death, particularly in cases of death without medical intervention and in the absence of an ascertained SARSCoV-2 infection and/or COVID-19 diagnosis, therefore providing support for diagnostic activity in the present COVID-19 pandemic. For this purpose, a standard operating procedure for correct swab collection, autopsy investigation and tissue sampling is provided.
\end{abstract}

Keywords SARS-CoV-2 $\cdot$ COVID-19 $\cdot$ Virological diagnosis $\cdot$ Autopsy investigation $\cdot$ Postmortem diagnosis

Hic est locus ubi mors gaudet succurrere vitae

"This is the place where death delights in helping life"

\section{Introduction}

SARS-CoV-2 (severe acute respiratory syndrome coronavirus 2) is a coronavirus responsible for COVID-19 (coronavirus disease 2019) which resulted in a cluster of cases of pneumonia that originated in China around 31 December 2019 and has subsequently spread across the globe. Currently, COVID-19 represents a health emergency worldwide, leading, in severe

Vittorio Fineschi

vfinesc@tin.it

1 Department of Anatomical, Histological, Forensic and Orthopaedic Sciences, Sapienza University of Rome, Viale Regina Elena 336, 00185 Rome, Italy

2 Department of Medicine, Surgery and Health, University of Trieste, Strada di Fiume 44, 34149 Trieste, Italy cases, to pneumonia, severe acute respiratory syndrome, multiorgan dysfunction or failure, and death [1].

Measures and recommendations have been developed to help healthcare personnel prevent the spread of the virus and to properly manage COVID-19 cases, advising on possible risks and preventive measures [2]. In the context of limited scientific knowledge and evidence of SARS-CoV-2 infection, guidance is becoming increasingly necessary for pathologists who have to carry out postmortem investigations on COVID19-related deaths [3-5].

The aim of the present report is to share a procedure for correct swab collection and autopsy investigations, offering technical support to professionals involved in these activities during the SARS-CoV-2 pandemic outbreak.

In each of the phases, the described procedure is carried out with the involvement of an operating pathologist and a support pathologist appointed to manage the samples and document the findings.

Such an approach can be used in cases of both suspected and probable COVID-19. The strength of this method is the development of a standard operating procedure for sampling and autopsy investigation in conditions of high biological risk. 


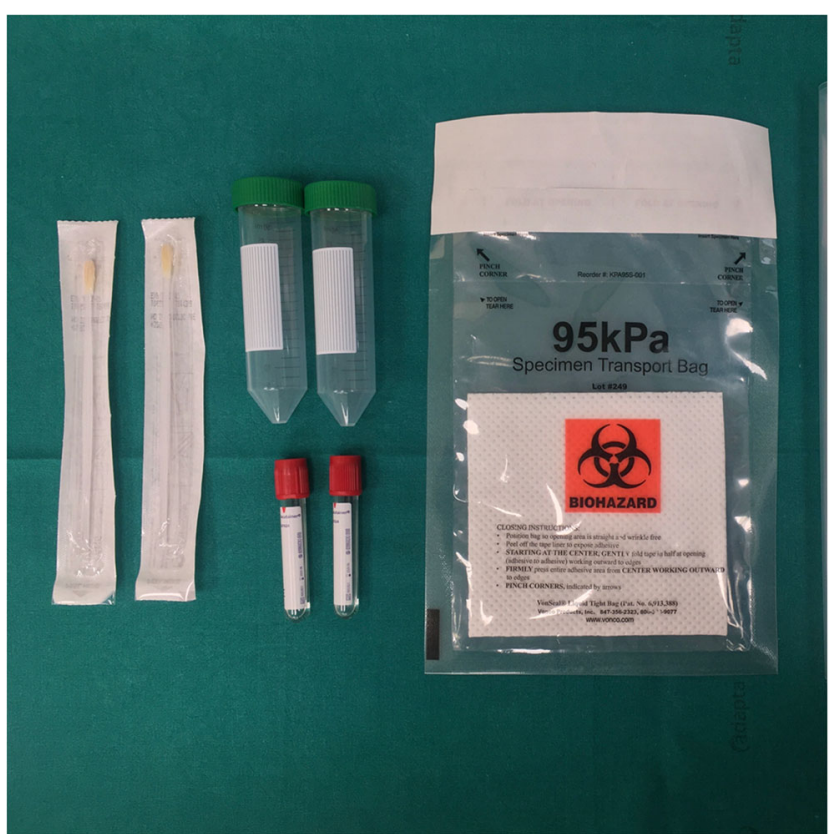

Fig. 1 Materials required for swabs collection

\section{Postmortem sampling for SARS-CoV-2 virological research}

The safe and correct sample collection for SARS-CoV-2 research by real-time PCR (RealStar ${ }^{\circledR}$ RT-PCR Altona Diagnostic) [6] requires the following materials (Fig. 1):

- 2 synthetic fiber swabs with plastic shafts;

- 2 sterile tubes (e.g. BD Vacutainer® $5 \mathrm{~mL}$ volume) containing 2-3 $\mathrm{mL}$ of viral transport medium or sterile saline;

- 2 Falcon ${ }^{\circledR}$ tubes with a volume of $50 \mathrm{~mL}$;

- 1 transparent plastic bag with adhesive closure and an absorbent pad for biohazard specimen transport;

- 1 transparent plastic bag with zip closure.

\section{Swab collection during external examination}

\section{Nasopharyngeal and oropharyngeal swabs}

Before the autopsy investigation, swabs of the upper respiratory tract (nasopharyngeal and oropharyngeal) are collected for virological SARS-CoV-2 research.

Collection of nasopharyngeal swabs is performed as follows (Fig. 2A):

1. insert the swab into the nostril, parallel to the palate;

2. leave the swab in place for a few seconds to absorb secretions;

3. perform a contralateral swab in the same manner and with the same device.

Collection of oropharyngeal swabs is performed as follows (Fig. 2B):

1. insert the swab into the oral cavity until it reaches the posterior pharyngeal wall (pay attention to avoiding contact with the tongue);

2. leave the swab in place for a few seconds to absorb secretions.

For safe sample preparation, after collection each of the swabs is placed in a sterile tube containing $2-3 \mathrm{~mL}$ of viral transport medium or sterile saline. The sterile tubes are then inserted into two different Falcon ${ }^{\circledR}$ tubes, which are placed into a plastic bag with adhesive closure and, subsequently, into a plastic bag with zip closure. Each container is labeled to indicate the identification number of the subject, the identification code of the sample, the type of sample and the date of collection.

Immediately after sampling, the swabs are sent to the laboratory for subsequent processing by real-time PCR.
Fig. 2 Collection of nasopharyngeal $\mathbf{a}$ and oropharyngeal b swabs
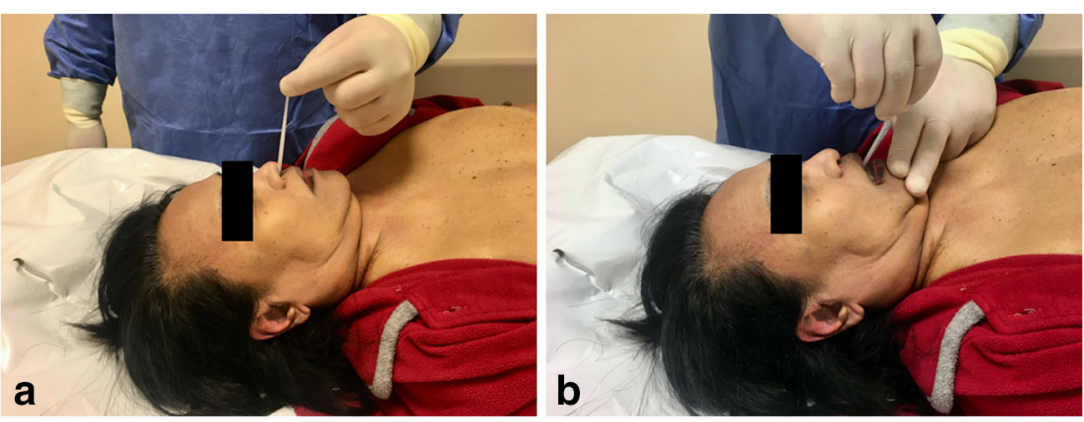


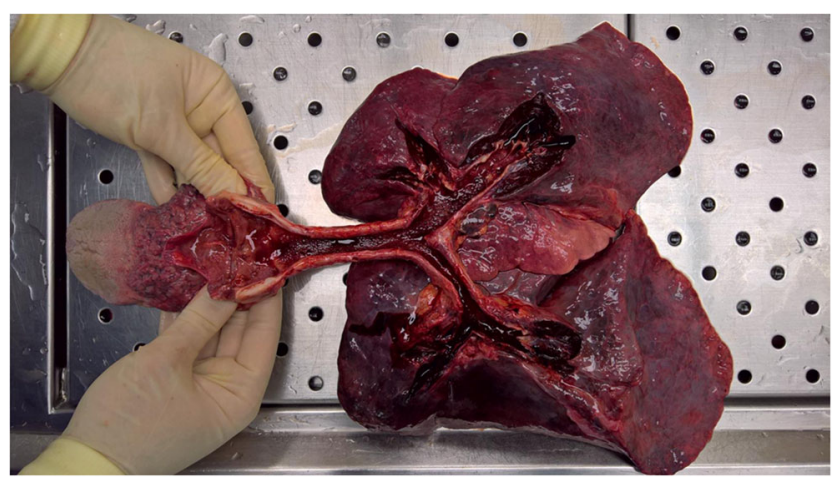

Fig. 3 Respiratory block (tongue-hypopharynx-larynx-trachea-bronchilungs) with a detailed view of airways

\section{Autopsy technique}

In the autopsy investigation of COVID-19 cases (suspected or probable) "en bloc" extraction (evisceration according to Ghon) of the cervicothoracic organs is performed.

According to this technique, "en bloc" extraction of the oral (tongue, oropharynx, hypopharynx), cervical (larynx, trachea) and thoracic (tracheal bifurcation, major bronchi, lungs) respiratory structures is performed, with final resection from the surrounding structures (aorta, inferior vena cava and esophagus) [7].

The basic steps for Ghon block removal are briefly described below:

1. the block is preliminarily prepared by dissecting the neck and chest muscles, removing the thyroid gland, opening the thoracic cavity by removing the sternocostal plate, and opening the pericardial sac;

2. the floor of the mouth is incised, making a "horseshoe incision" from one mandibular corner to the other, gaining access to the oral cavity and permitting visualization of the base of the tongue;

Fig. 4 Collection of bilateral lower respiratory tract swabs (ab)
3. the block of tongue-hypopharynx-esophagus-larynxtrachea is isolated from the vertebral-cervical segment by dissecting the root of the tongue and hypopharynx and then proceeding with sectioning in the axis of the vertebral column in a proximo-distal direction;

As an alternative to steps 2 and 3 , the cervical block can be dissected above the thyroid cartilage to avoid the risk of sharp injury and to facilitate the maneuver for less experienced operators.

4. cervical-thoracic evisceration is completed by dissecting the aortic branch vessels from the thoracic outlet, cutting in the vertebral plane until the diaphragm is reached, overturning the block onwards and down onto the diaphragm, isolating the esophagus, transversally cutting the descending aorta slightly further from its origin, and finally cutting the inferior vena cava and the pericardial sac along the fusion line with the diaphragm;

5. the obtained cardiovascular-respiratory block is positioned on its anterior surface: the aorta is separated from the pulmonary trunk, the pulmonary arteries are opened from the trunk towards the pulmonary hilum, the pulmonary veins are resected near the hilum, the aortic branch vessels are separated from the trachea and the bronchi, and finally, the cardio-aortic block is isolated;

6. the obtained respiratory block (tongue-hypopharynx-larynx-trachea-bronchi-lungs) is positioned on its posterior surface: the larynx is opened along the posterior median line, while the trachea and the principal bronchi are opened along their posterior membranous walls (Fig. 3).
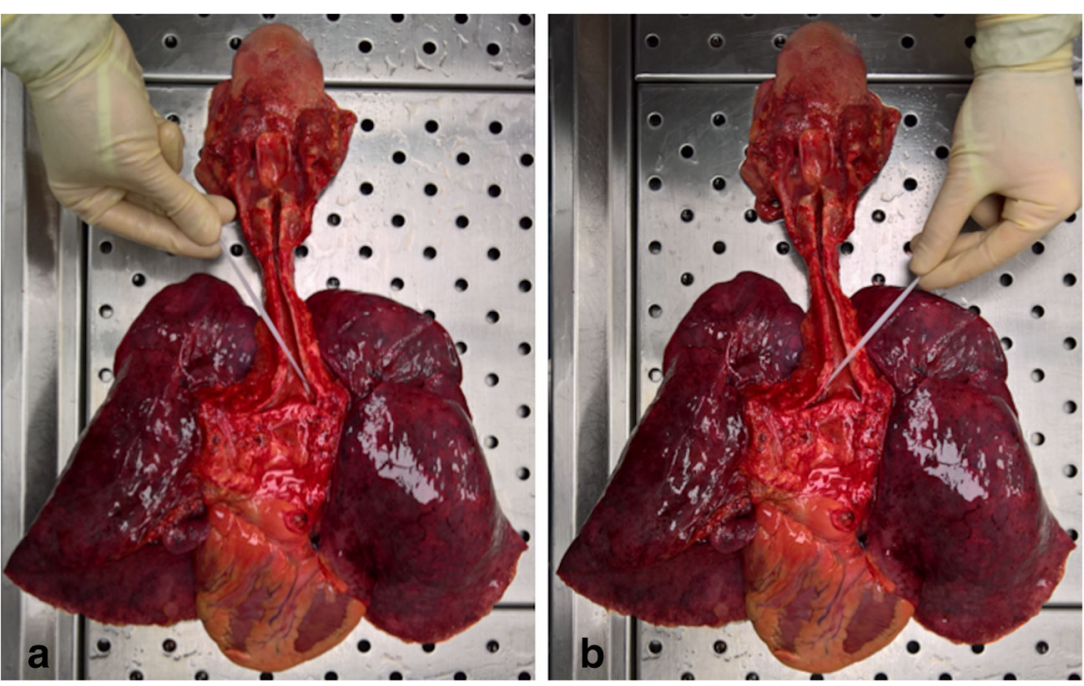
Fig. 5 Diagnostic algorithm for postmortem sampling and

autopsy investigation in cases of suspected or probable COVID-19

\section{Diagnostic algorithm for postmortem sampling and \\ autopsy investigation in cases of suspected or probable COVID-19}

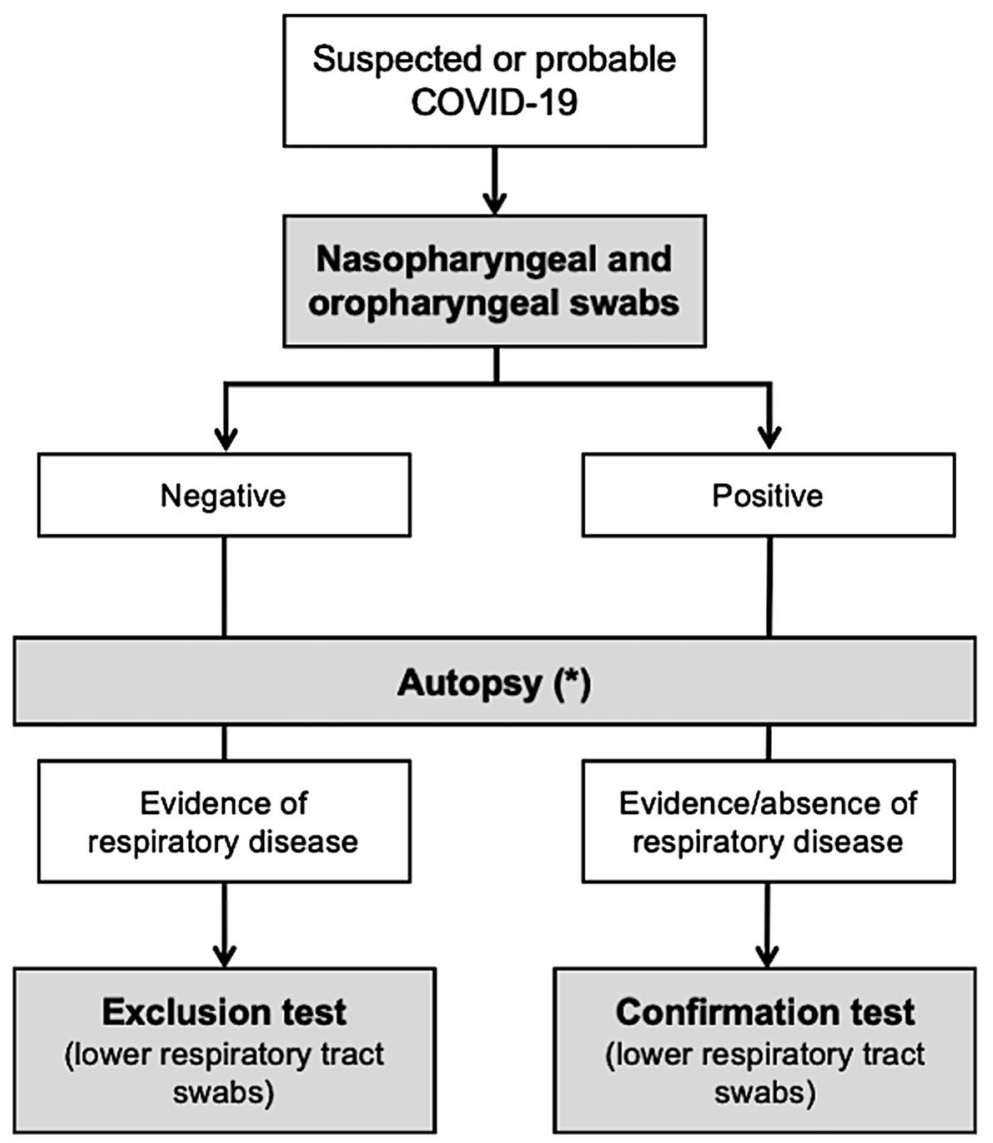

${ }^{*}$ If nasopharyngeal and oropharyngeal swabs are negative, the autopsy is performed in accordance with the protocols for sudden death.

If nasopharyngeal and oropharyngeal swabs are positive, the autopsy is performed by extraction "en bloc" of the cervicothoracic organs, with standard sampling of trachea (proximal and distal), primary bronchi, and lungs (one sample for each lobe and a sample of the hilar region bilaterally).

\section{Swab collection during autopsy investigation}

Lung swabs

Intra-autopsy swab collection for virological SARS-CoV-2 research is performed after the removal and opening of the respiratory block from the lower respiratory tract (primary bronchi).
Collection of the lung swabs is performed as follows:

1. insert the swab into the branches of the main bronchus (Fig. 4A);

2. leave the swab in place for a few seconds to absorb secretions;

3. perform a contralateral swab in the same manner but with a different device (Fig. 4B). 
For safe sample preparation for transport and processing, the procedure is the same as that mentioned above for the nasopharyngeal and oropharyngeal swabs.

\section{Tissue sampling for histology and immunohistochemistry}

Standard samples collected for histopathological investigation of COVID-19 cases include the following:

- Respiratory system: trachea (proximal and distal); right and left primary bronchi; hilar lung with segmental bronchi (bilaterally); representative pulmonary parenchyma from the right and left lungs (one sample for each lobe);

- Additional samples: brain, heart, liver, spleen, kidney, gastrointestinal tract and any other tissue with macroscopically evident pathological involvement.

\section{Discussion}

The present procedure was designed according to a step-bystep diagnostic approach (Fig. 5). In particular, postmortem sampling for SARS-CoV-2 virological research by real-time PCR is always carried out in cases of a decedent with suspected or probable COVID-19 in two steps:

1. During external examination, nasopharyngeal and oropharyngeal swabs are collected to assess and label the cases as SARS-CoV-2 positive or negative (SARS-CoV2 detected or not detected).

2. During autopsy, as an adjunct, swab collection from the primary bronchi is performed in the following cases:

- positive pre-autopsy nasopharyngeal/oropharyngeal swab (SARS-CoV-2 detected) with or without significant autopsy pulmonary findings, suggestive of lung disease (confirmation test);

- negative pre-autopsy nasopharyngeal/oropharyngeal swab (SARS-CoV-2 not detected) with significant autopsy pulmonary findings, suggestive of lung disease (exclusion test).

For this purpose, a complete autopsy with extraction of the Ghon respiratory block is therefore of considerable importance, as it allows preservation of the anatomical integrity of the upper and lower airways and correct sampling of the primary bronchi for virological confirmation/exclusion.

The correct visualization of the whole respiratory tract, as well as the description and evaluation of its macroscopic characteristics (caliber, width, intraluminal content), are also facilitated. Furthermore, the advantages of the described technique include the possibility of proceeding with complete and accurate sampling, which is useful for a definitive pathological diagnosis through histological and immunohistochemical investigations [8-11].

In conclusion, the present procedure is applicable in cases of COVID-19-related death, particularly in cases of death without medical intervention and in the absence of an ascertained SARS-CoV-2 infection and/or COVID-19 diagnosis, therefore providing support and an approach for the diagnostic activity in the present COVID-19 pandemic.

Authors contribution All the authors contributed equally to the conception and design of the study, acquisition of data, or analysis and interpretation of data, drafted and revised the article critically for important intellectual content, and approved the final version before its submission.

Funding information Authors research was not supported and funded.

\section{Compliance with ethical standards}

Conflict of interest Authors and corresponding author don't have conflict of interests in writing and submitting for publication the manuscript.

Code availability Not applicable.

\section{References}

1. Weiss P, Murdoch DR. Clinical course and mortality risk of severe COVID-19. Lancet. 2020;395:1014-5.

2. European Centre for Disease Prevention and Control. Case definition and European surveillance for COVID-19, as of 2 March 2020. www.ecdc.europa.eu/en/case-definition-and-europeansurveillance-human-infection-novel coronavirus-2019-ncov. Accessed 1 April 2020.

3. Fineschi V, Aprile A, Aquila I, Arcangeli M, Asmundo A, Bacci M, et al. Management of the corpse with suspect, probable or confirmed COVID-19 respiratory infection - Italian interim recommendations for personnel potentially exposed to material from corpses, including body fluids, in morgue structures and during autopsy practice. Pathologica. 2020. https://doi.org/10.32074/ 1591-951X-13-20.

4. Centers for Disease Control and Prevention. Interim Guidance for Collection and Submission of Postmortem Specimens from Deceased Persons Under Investigation (PUI) for COVID-19, February 2020. https://www.cdc.gov/coronavirus/2019-ncov/hcp/ guidance-postmortem-specimens.html\#SpecimenCollection. Accessed 1 April 2020.

5. World Health Organization. Laboratory testing for coronavirus disease 2019 (COVID-19) in suspected human cases. 2020. https:// www.who.int/publications-detail/laboratory-testing-for-2019novel-coronavirus-in-suspected-human-cases-20200117. Accessed 1 April 2020.

6. Corman VM, Landt O, Kaiser M, Molenkamp R, Meijer A, Chu DKW, et al. Detection of 2019 novel coronavirus (2019-nCoV) by real-time RT-PCR. Eurosurveillance. 2020;25:23.

7. Pomara C, Karch SB, Fineschi V. The autopsy. In: Pomara C, Karch SB, Fineschi V, editors. Forensic autopsy - a handbook and atlas. Boca Raton: CRC Press; 2010. 
8. Xu Z, Shi L, Wang Y, Zhang J, Huang L, Zhang C, et al. Pathological findings of COVID-19 associated with acute respiratory distress syndrome. Lancet Respir Med. 2020;8:420-2.

9. Luo W, Yu H, Gou J, Li X, Sun Y, Li J, et al. Clinical pathology of critical patient with novel coronavirus pneumonia (COVID-19). Preprints; 2020:2020020407.

10. Lucas S. Autopsies on people with high-risk infections. In: Burton JL, Rutty G, editors. The hospital autopsy: a manual of fundamental autopsy practice. 3rd ed. London: Hodder Arnold; 2010.
11. The Royal College of Pathologists. Briefing on COVID-19. Autopsy practice relating to possible cases of COVID-19 (2019nCov, novel coronavirus from China 2019/2020). https://www. rcpath.org/uploads/assets/d5e28baf-5789-4b0f-acecfe370eee6223/ fe8fa85a-f004-4a0c-81ee4b2b9cd12cbf/Briefing-on-COVID-19autopsy-Feb-2020.pdf. Accessed 1 April 2020.

Publisher's note Springer Nature remains neutral with regard to jurisdictional claims in published maps and institutional affiliations. 